International Journal of Pure and Applied Mathematics

Volume 94 No. 5 2014, 661-667

ISSN: 1311-8080 (printed version); ISSN: 1314-3395 (on-line version)

url: http://www.ijpam.eu

doi: http://dx.doi.org/10.12732/ijpam.v94i5.3

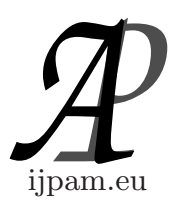

\title{
EQUITABLE DOMINATION IN FUZZY GRAPHS
}

\author{
K.M. Dharmalingam ${ }^{1}$, M. Rani ${ }^{2} \S$ \\ ${ }^{1}$ Department of Mathematics \\ The Madura College \\ Madurai, Tamilnadu State, INDIA \\ ${ }^{2}$ Department of Mathematics \\ Madurai Kamaraj University College \\ Aundipatti, Tamilnadu State, INDIA
}

\begin{abstract}
Let $G$ be a fuzzy graph. Let $u$ and $v$ be two vertices of $G$. A subset $D$ of $V$ is called a fuzzy equitable dominating set if every $v \in V-D$ there exist a vertex $u \in D$ such thatuv $\in E(G)$ and $|\operatorname{deg}(u)-\operatorname{deg}(v)| \leq 1$ where $\operatorname{deg}(u)$ denotes the degree of vertex $u$ and $\operatorname{deg}(v)$ denotes the degree of vertex $v$ and $\mu(u v) \leq \sigma(u) \wedge \sigma(v)$. The minimum cardinality of a fuzzy equitable dominating set is denoted by $\gamma^{e f}$. In this paper we introduce the concept of fuzzy equitable dominating set, minimal fuzzy equitable dominating set, strong (weak) fuzzy equitable dominating set, fuzzy equitable independent set and obtain some interesting results for this new parameter in equitable fuzzy graphs.
\end{abstract}

AMS Subject Classification: $05 \mathrm{C} 72$

Key Words: fuzzy equitable dominating set, fuzzy equitable neighborhood, fuzzy equitable independent set

\section{Introduction}

L.A. Zadeh (1965) introduced the concepts of a fuzzy subset of a set as a way for representing uncertainty. His idea have been applied to a wide range of scientific areas.

Fuzzy concepts is also introduced in Graph theory. Formally, a fuzzy graph $G=(V, \sigma, \mu)$ is a non empty set $V$ together with a pair of functions

Received: February 10, 2014

(c) 2014 Academic Publications, Ltd.

$\S$ Correspondence author url: www.acadpubl.eu 
$\sigma: V \rightarrow[0,1]$ and $\mu: V \times V \rightarrow[0,1]$ such that $\mu(u v) \leq \sigma(u) \wedge \sigma(v)$ for all $u, v$ in $V, \sigma$ is called the fuzzy vertex set of $G$ and $\mu$ is called the fuzzy edge set of $G$. The concept of equitable domination [11] in graphs was introduced by Venkatrasubramanian Swaminathan and Kuppusamy Markandan Dharmalingam. The notation of domination in fuzzy graphs [9] was developed by A. Somasundaram and S.Somasundaram. In this paper we introduce the new concepts in equitable domination in fuzzy graphs.

\section{Preliminaries}

Definition 1.1. A fuzzy graph $G=(\sigma, \mu)$ is a set with two functions $\sigma: \mathrm{V} \rightarrow[0,1]$ and $\mu: V \times V \rightarrow[0,1]$ such that $\mu(u v) \leq \sigma(u) \wedge \sigma(v)$ for all $u, v \in V$.

Definition 1.2. Let $G=(\sigma, \mu)$ be a fuzzy graph on $V$ and $V_{1} \subseteq V$. Define $\sigma_{1}$ on $V_{1}$ by $\sigma_{1}(u)=\sigma(u)$ for all $u \in V_{1}$ and $\mu_{1}$ on the collection $E_{1}$ of two element subset of $V_{1}$ by $\mu_{1}(u, v)=\mu(u, v)$ for all $u, v \in V_{1}$. Then $\left(\sigma_{1}, \mu_{1}\right)$ is called the fuzzy subgraph of $G$ induced by $V_{1}$ and is denoted by $\left\langle V_{1}\right\rangle$.

Definition 1.3. The order $p$ and size of $q$ of a fuzzy graph $G=(\sigma, \mu)$ are defined to be $p=\sum_{u \in V} \sigma(u)$ and $q=\sum_{u v \in E} \mu(u v)$.

Definition 1.4. The degree of vertex $u$ is defined as the sum of the weights of the edges incident at $u$ and is denoted by $\operatorname{deg}(u)$.

\section{Definitions and Main Results}

Definition 1.5. Let $G$ be a fuzzy graph. Let $u$ and $v$ be two vertices of $G$. A subset $D$ of $V$ is called a fuzzy equitable dominating set if for every $v \in V-D$ there exist a vertex $u \in D$ such that $u v \in E(G)$ and $|\operatorname{deg}(u)-\operatorname{deg}(v)| \leq 1$ and $\mu(u v) \leq \sigma(u) \wedge \sigma(v)$. The minimum cardinality of a fuzzy equitable dominating set is denoted by $\gamma^{e f}$. 


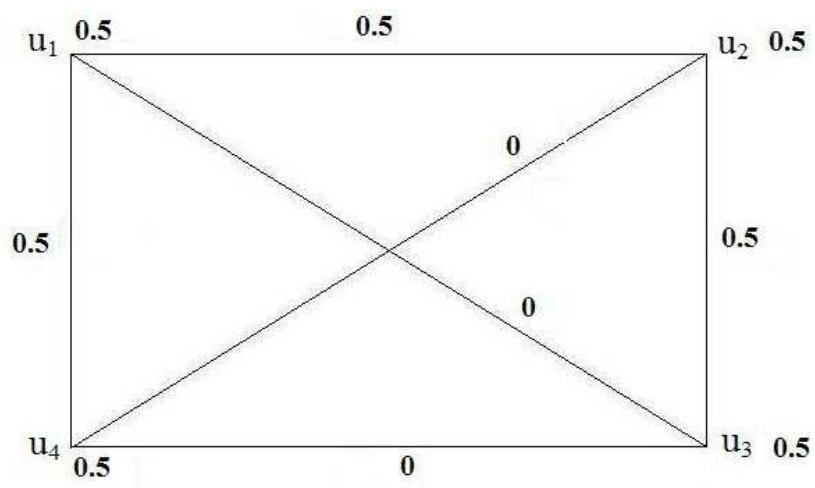

Figure 1

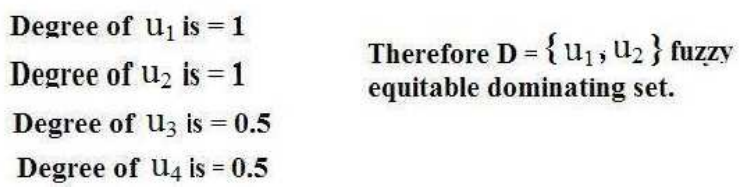

Definition 1.6. A vertex $u \in V$ is said to be degree equitable fuzzy graph with a vertex $v \in V$ if $|\operatorname{deg}(u)-\operatorname{deg}(v)| \leq 1$ and $\mu(u v) \leq \sigma(u) \wedge \sigma(v)$.

Definition 1.7. If $D$ is an fuzzy equitable dominating set then any super set of $D$ is a fuzzy equitable dominating set.

Definition 1.8. A fuzzy equitable dominating set $D$ is said to be a minimal fuzzy equitable dominating set if no proper subset of $D$ is a fuzzy equitable dominating set.

Theorem 1.1. A dominating set $D$ of $G$ is a minimal fuzzy equitable dominating set iff for each $d \in D$ one of the following two conditions holds:

i) $N^{e f}(d) \cap D=\phi$

ii) there is a vertex $c \in V-D$ such that $N^{e f}(c) \cap D=\{d\}$.

Proof. Let $D$ be a minimal fuzzy equitable dominating set and $d \in D$. Then $D_{d}=D \backslash\{d\}$ is not a fuzzy equitable dominating set and hence there exists $x \in V \backslash D_{d}$ such that $\mathrm{x}$ is not dominated by any element of $D_{d}$. If $x=d$ we get (i) and if $x \neq d$ we get (ii). The converse is obvious.

Definition 1.9. Let $u \in V$. The fuzzy equitable neighbourhood of $u$ denoted by $N^{e f}(u)$ is defined as

$N^{e f}(u)=\{v \in V|v \in N(u),| \operatorname{deg}(u)-\operatorname{deg}(v) \mid \leq 1$ and $\mu(u v) \leq \sigma(u) \wedge \sigma(v)\}$ and $u \in I_{e} \Longleftrightarrow N^{e f}(u)=\phi$.

The cardinality of $N^{e f}(u)$ is called fuzzy equitable degree of $u$ and it is denoted by $d_{G}^{e f}(u)$. 
Definition 1.10. The maximum and minimum fuzzy equitable degree of a vertex in $G$ are denoted respectively by $\Delta^{e f}(G)$ and $\delta^{e f}(G)$. That is $\Delta^{e f}(G)=$ $\max _{u \in V(G)}\left|N^{e f}(u)\right|$ and $\delta^{e f}(G)=\min _{u \in V(G)}\left|N^{e f}(u)\right|$.

Definition 1.11. Let $G$ be a fuzzy graph. Then $D \subseteq V$ is said to be a strong (weak) fuzzy equitable dominating set of $G$ if every vertex $v \in V-D$ is strongly (weakly) dominated by some vertex $u$ in $D$. We denote a strong (weak) fuzzy equitable dominating set by sefd-set (wefd-set).

The minimum scalar cardinality of a sefd-set (wefd-set) is called the strong (weak) fuzzy equitable domination number of $G$ and it is denoted by $\gamma^{\text {sef }}(G)$ $\left(\gamma^{w e f}(G)\right)$.

Theorem 1.2. Let $G$ be a fuzzy graph of order $p$, then:

i) $\gamma^{e f}(G) \leq \gamma^{\text {sef }}(G) \leq p-\Delta^{e f}(G)$,

ii) $\gamma^{e f}(G) \leq \gamma^{\text {wef }}(G) \leq p-\delta^{e f}(G)$.

Proof. Every strong fuzzy equitable dominating set is a equitable dominating set of $\mathrm{G}, \gamma^{e f}(G) \leq \gamma^{\text {sef }}(G)$ and every weak fuzzy equitable dominating set is a equitable dominating set of $\mathrm{G}, \gamma^{e f}(G) \leq \gamma^{\text {wef }}(G)$. Let $u, v \in V$. If $d_{G}^{e f}(u)=\Delta^{e f}(G)$ and $d_{G}^{e f}(v)=\delta^{e f}(G)$ clearly $V-N^{e f}(u)$ is a strong fuzzy equitable dominating set and $V-N^{e f}(v)$ is a weak fuzzy equitable dominating set. Therefore $\gamma^{\text {sef }}(G) \leq\left|V-N^{e}(u)\right|^{e f}$ and $\gamma^{\text {wef }}(G) \leq\left|V-N^{e}(v)\right|^{e f}$ i.e $\gamma^{\text {sef }}(G) \leq p-\Delta^{e f}(G)$ and $\gamma^{\text {wef }}(G) \leq p-\delta^{e f}(G)$.

Definition 1.12. A vertex $u$ of a equitable fuzzy graph is said to be an equitable isolated vertex if a vertex $u \in V$ be such that $|\operatorname{deg}(u)-\operatorname{deg}(v)| \geq 2$ and if $\mu(u v) \leq \sigma(u) \wedge \sigma(v)$ for all $v \in V-\{u\}$ i.e $N^{e f}(u)=\phi$.

Theorem 1.3. Let $G$ be a fuzzy graph without isolated vertices. Let $D$ be a minimal fuzzy equitable dominating set of $G$. Then $V \backslash D$ is a fuzzy equitable dominating set of $G$.

Proof. Let $\mathrm{d}$ be any vertex in D. Since G has no isolated vertices. There is a vertex $c \in N(d)$. It follows from Theorem 1.1 that $c \in V \backslash D$. Thus every element of $\mathrm{D}$ is dominated by some element of $V \backslash D$.

\section{Equitable Independent Domination in Fuzzy Graphs}

Definition 2.1. A set $S$ of vertices of a fuzzy graph is said to be fuzzy equitable independent set, if for any $u \in S, v \notin N^{e}(u)$ for all $v \in S-\{u\}$ and $\mu(u v) \leq \sigma(u) \wedge \sigma(v) \forall u, v \in S$. 
Theorem 2.1. If $D$ is a fuzzy equitable independent dominating set of a graph $G$ then $D$ is a both minimal fuzzy equitable dominating set and a maximal fuzzy equitable independent set. Conversely any maximal fuzzy equitable independent set $D$ in $G$ is a fuzzy equitable independent dominating set of $G$.

Proof. If $D$ is a fuzzy equitable independent dominating set of $G$. $D_{d}=$ $D \backslash\{d\}$ is not a fuzzy equitable dominating set for every $d \in D$ and $D \cup\{x\}$ is not a fuzzy equitable independent for every $x \notin D$ so that $D$ is a minimal fuzzy equitable dominating set and a maximal fuzzy equitable independent set.

Conversely, let $D$ be a maximal fuzzy equitable independent set in $G$. Then for every $x \in V \backslash D, D \cup\{x\}$ is not fuzzy equitable independent and hence $x$ is dominated by some element of $D$. Thus $D$ is a fuzzy equitable independent dominating set of $G$.

Theorem 2.2. Let $G$ be a fuzzy graph. Then $\gamma^{e f} \leq p-\triangle^{e f}$.

Proof. Let $\mathrm{v}$ be vertex such that $d_{G}^{e f}(v)=\triangle^{e f} \operatorname{Then} V \backslash N^{e f}(v)$ is a fuzzy equitable dominating set of G so that $\gamma^{e f} \leq\left|V \backslash N^{e f}(v)\right|=p-\triangle^{e f}$.

Theorem 2.3. Let $G$ be a fuzzy graph. Then $\gamma^{e f}(G) \leq \beta_{0}^{e f}(G)$.

Proof. Let $\mathrm{S}$ be a fuzzy equitable independent set of vertices in $\mathrm{G}$ such that $|S|=\beta_{0}^{e f}(G)$. Then $\mathrm{G}$ contains no large fuzzy equitable independent set. This means that every vertex $\mathrm{v}$ in $\mathrm{V}-\mathrm{S}$ is adjacent to atleast one vertex of $\mathrm{S}$. Therefore $\mathrm{S}$ is a fuzzy equitable dominating set. Thus $\gamma^{e f}(G) \leq|S|$. Therefore $\gamma^{e f}(G) \leq \beta_{0}^{e f}(G)$.

Theorem 2.4. A fuzzy equitable independent set $S$ is maximal fuzzy equitable independent set iff it is fuzzy equitable independent set and fuzzy equitable dominating set.

Proof. Suppose a fuzzy equitable independent set $S$ is maximal fuzzy equitable independent set. Then for every vertex $u$ in $V-S$, the set $S \cup\{u\}$ is not fuzzy equitable independent set, that is for very vertex $u$ in $V-S$ there is a vertex $v$ in such that $u$ is adjacent to $v$. Thus $S$ is a fuzzy equitable dominating set. Hence $S$ is both fuzzy equitable independent and fuzzy equitable dominating set.

Conversely suppose that a set $S$ is both fuzzy equitable independent and fuzzy equitable dominating set. We show that it is maximal fuzzy equitable independent set. Suppose $S$ is not maximal fuzzy equitable independent set. Then there exist a vertex $u$ in $V-S$ such that $S \cup\{u\}$ is a fuzzy equitable independent set. But if $S \cup\{u\}$ is a fuzzy equitable independent set, then no vertex in $S$ is adjacent to $u$. Hence $S$ is not a fuzzy equitable dominating set, 
which is a contradiction. Therefore $S$ is maximal fuzzy equitable independent set.

\section{Conclusion}

For graphical research the fuzzy equitable domination and fuzzy equitable independent are very useful for solving wide range of problems. More than fifty domination parameters have been investigated by different authors. The membership functions $\sigma$ and $\mu$ on the vertex set and the edge set of $\mathrm{G}$ can be constructed from the Statistical data that represents the number of vehicles passing through various junctions and the number of vehicles passing through various roads during a peak hour. This paper we have introduced the concepts of fuzzy equitable domination and fuzzy equitable independent. Additional new concept of fuzzy equitable domination will be reported in forth coming paper.

\section{References}

[1] E.J.Cockayne and S.T. Hedetniemi, Towards a theory of domination in graphs, Networks. (1977), 247-261

[2] T.W Haynes et. al. Fundamentals of Domination in Graphs, Marcel Dekker, New York,1998

[3] Kuppusamy Markandan Dharmalingam, Equitable Associate Graph of a Graph, Bulletin of International Mathematical Virtual Institue 2(1)(2012) $109-116$

[4] J.N Mordeson and P.S Nair, Fuzzy graphs and Fuzzy Hyper graphs, Physica Verlag, Heidelberg, 1998, Second edition 2001

[5] A. Nagoor Gani and V.T. Chandra Sekaran, Domination in Fuzzy Graph, Advances in Fuzzy Sets and Systems, 1(1)(2006), 17-26

[6] A. Nagoor Gani, P.Vadivel, On Domination, Independence and Iredundance in Fuzzy Graph, International Review of Fuzzy Mathematics, Volume 3, No 2, (June 2008) 191-198

[7] A. Nagoor Gani, P. Vadivel, Contribution to the theory of Domination, Independence and Irredundance in Fuzzy graph, Bulletin of Pure and Applied Sciences, Vol-28E (No-2) 2009, 179-187 
[8] A Rosenfield, Fuzzy graphs, in, L.A.Zadeh, K.S. Fu, M.Shimura (Eds), Fuzzy sets and their Applications to congnitive and Decision Processes, Academic Press, New York, 1975, 77-95

[9] A. Somasundaram and S.Somasundaram, Domination in Fuzzy Graphs-I, Elsevier Science, 19(1998), 787-791

[10] E. Sampath Kumar, L.Puspalatha, Strong, weak domination and domination balance in a graph, Discrete Math. 161(1966) 235 - 242

[11] Vekatasubramanian Swaminathan and Kuppusamy Markandan Dharmalingam, Degree Equitable Domination on Graphs, Kragujevac Journal of Mathematics, Volume 35, Number 1, PP 177-183, (2011). 
